\title{
Inhibition of autophagy enhances anticancer effects of bevacizumab in hepatocarcinoma
}

\author{
Xian-ling Guo • Ding Li • Kai Sun • Jin Wang • Yan Liu • \\ Jian-rui Song • Qiu-dong Zhao • Shan-shan Zhang • \\ Wei-jie Deng • Xue Zhao $\cdot$ Meng-chao Wu $\cdot$ Li-xin Wei
}

Received: 19 February 2012 /Revised: 19 September 2012 / Accepted: 28 September 2012 /Published online: 10 October 2012

(C) The Author(s) 2012. This article is published with open access at Springerlink.com

\begin{abstract}
Angiogenesis inhibitors have long been considered desirable anticancer agents. However, it was found that many tumors could develop resistance to antiangiogenesis inhibitors. Antiangiogenic therapy results in metabolic stress. Autophagy is an important survival mechanism in cancer cells under metabolic stress; however, it remains unknown if autophagy contributes to antiangiogenesis resistance. In this study, we reported that bevacizumab treatment reduced the development of new blood vessels and inhibited cell growth in xenografts of hepatocellular carcinoma (HCC) tumors. Bevacizumab treatment also upregulated expression of the autophagy-related genes (Beclin1 and LC3) and increased autophagosome formation. Our in vitro studies demonstrated that autophagy inhibition significantly increased apoptosis of HCC cells during nutrient starvation or hypoxia. In addition, the combined treatment of an autophagy inhibitor and bevacizumab markedly inhibited the tumor growth of HCC
\end{abstract}

Electronic supplementary material The online version of this article (doi:10.1007/s00109-012-0966-0) contains supplementary material, which is available to authorized users.

Xian-ling Guo, Ding Li, and Kai Sun contributed equally to this work.

X.-l. Guo · D. Li $\cdot$ K. Sun · Y. Liu · J.-r. Song • Q.-d. Zhao •

S.-s. Zhang $\cdot$ W.-j. Deng $\cdot$ X. Zhao $\cdot$ M.-c. Wu $\cdot$ L.-X. Wei $(\bowtie)$

Tumor Immunology and Gene Therapy Center, Eastern

Hepatobiliary Surgery Hospital,

The Second Military Medical University,

225 Changhai Road,

Shanghai 200438, People's Republic of China

e-mail: weilixin@yahoo.com

\section{X.-1. Guo}

Hang Zhou Sanitarium of Navy,

Zhejiang, People's Republic of China

J. Wang

Department of Oncology, Chang Zheng Hospital,

The Second Military Medical University,

Shanghai, People's Republic of China xenografts, led to enhanced apoptosis, and impaired the proliferation of tumor cells compared with treatment with either drug alone. Furthermore, autophagy inhibition led to enhanced reactive oxygen species (ROS) generation in HCC cells exposed to nutrient starvation or hypoxia in vitro and increased DNA oxidative damage in vivo. Antioxidants reduced nutrient starvation or the hypoxia-induced cell death of HCC cells after autophagy inhibition. Our results suggest that autophagy modulates ROS generation and contributes to cell survival under metabolic stress. Therefore, autophagy inhibition may be a novel way of increasing the efficicacy of antiangiogenic agents in the treatment of HCC.

Keywords Hepatocarcinoma - Antiangiogenesis · Autophagy $\cdot$ Metabolic stress $\cdot$ Apoptosis

\section{Introduction}

Hepatocarcinoma (HCC) is a hypervascular tumor characterized by neovascularization. Its growth relies on the formation of new blood vessels [1]. Vascular endothelial growth factor (VEGF) expression is upregulated in HCC compared with the cirrhotic or normal liver $[2,3]$. These findings strongly suggest that antiangiogenic strategy may be therapeutically beneficial in the management of HCC. The effects of several antiangiogenic agents, including bevacizumab, have been evaluated in preclinical studies. Bevacizumab is a recombinant, humanized monoclonal antibody that binds VEGF with high affinity and has been approved by the FDA for treatment of certain late-stage cancers $[4,5]$. Several studies have explored the clinical efficacy of bevacizumab in patients with advanced HCC; however, its therapeutic effects have not been satisfied [6].

Autophagy is an evolutionarily conserved mechanism that exists in all eukaryotes from yeast to mammals [7]. During autophagy, a double membrane, known as the isolation 
membrane, wraps around portions of the cytoplasm and intracellular organelles to form a double-membrane vesicle called autophagosome. Upon maturation, autophagosomes fuse with lysosomes to form autolysosomes and are degraded by lysosomal proteases $[8,9]$. In addition to its homeostatic role, the autophagic process allows cells to survive nutrient stress, by providing amino acids and fatty acids to maintain metabolic levels. The genes that regulate autophagy were first identified in yeasts, many of which have homologues in higher eukaryotes [10]. Among them, LC3 [11] and Beclin1 [12] are widely used as markers of autophagy.

Antiangiogenic therapy leads to nutrient deprivation and oxygen stress in solid tumors; autophagy helps cells cope with these unfavorable conditions. Therfore, we hypothesized that autophagy inhibition could enhance the therapeutic efficacy of antiangiogenic treatments. To test this, we investigated the effect of bevacizumab on autophagy and determined if autophage inhibition could enhance the sensitivity of hepatocarcinoma to bevacizumab treament.

\section{Materials and methods}

\section{Cell culture and reagents}

The human hepatocarcinoma cell lines, SMMC-7721 and Hep3B, were maintained in Dulbecco's modified Eagle's medium (DMEM) (GIBCO, Invitrogen) and supplemented with $10 \%$ fetal bovine serum at $37{ }^{\circ} \mathrm{C}$ in a humidified $5 \% \mathrm{CO}_{2}$ incubator. Nutrient deprivation was carried out in Earle's balanced salts (EBSS) medium (Sigma-Aldrich). 3-Methyladenine (3-MA), chloroquine (CQ), and $N$-acetyl-cysteine (NAC) were obtained from Sigma-Aldrich (Shanghai, China). Bevacizumab was obtained from Roche (Shanghai, China).

Cell viability assay

The measurement of viable cell mass was assessed by a Cell Counting Kit (Dojin Laboratories, Kumamoto, Japan), as previously described [13].

Western blot analysis

Western blot analysis was performed as described [14]. Antibodies used were specific for Beclin1, LC3, HIF-1 $\alpha$, and GAPDH (Epitomics, Inc.).

Immunohistochemical analysis

Excised tumor tissue specimens from nude mice were formalin-fixed and embedded in paraffin. Sections 3-5 $\mu \mathrm{m}$ thick were cut ( $n=4$ tumor sections from each tumor), mounted, and stained with the following antibodies: rabbit
anti-CD31 (1:50; Bioworld Technology), rabbit anti-PCNA (1:2,000; Bioworld Technology), rabbit anti-activated caspase-3 (1:1,000; Bioworld Technology), rabbit antiBeclin1 (1:200; Novus Biologicals), rabbit anti-LC3 (1:200; Novus), mouse anti-HIF-1 $\alpha$ (1:100; Santa Cruz), and goat anti- 8 hydroxyguanosine antibody $(1: 400$, Abcam). Horseradish peroxidase (HRP)-labeled goat antirabbit immunoglobulin and HRP-labeled goat anti-mouse immunoglobulin (Santa Cruz) were applied as secondary antibodies for $30 \mathrm{~min}$ at $37^{\circ} \mathrm{C}$, followed by the streptavidin-biotin complex method. Immunostaining was developed with DAKO Liquid DAB +Substrate-Chromogen System (Changdao BioTech), followed by counterstaining with hematoxylin. PCNA-positive nuclei were counted in five random fields per tumor $(400 \times)$ and were expressed as a percentage of the total number of cells observed in each individual tumor. Activated caspase-3-positive cells were counted in five random fields per tumor $(200 \times)$. Data were expressed as mean \pm standard deviation (SD). Tumor tissue images were taken using the LEICA DM RXA2 microscope.

\section{TUNEL assay}

Analysis of apoptotic cells in tumor tissue was performed by terminal deoxynucleotidyl transferase-mediated dUTP nickend labeling (TUNEL) staining using the apoptotic cell detection kit following the manufacturer's directions (Merck Corp.). TUNEL-positive (apoptosis) cells had a pyknotic nucleus with dark green fluorescent staining. Each sample was observed at a magnification of $400 \times$, and TUNEL-positive cells were calculated in five random fields per tumor.

siRNA oligonucleotides and recombinant adenovirus

Stealth RNAiTM siRNA duplex oligoribonucleotides were used as siRNAs to human Beclin1 [14]. The production of recombinant adenovirus (Adsi-Beclin1) was performed as previously described [15]. The synthesized oligonucleotides against Beclin1 were: forward, 5'-GATCCCCCAGTTTGGCACAATCAA TATTCAAGAGATATTGATTGTGCCAAACTGTTTTTA-3' and reverse, 5'-AGCTTAAAAACAGTTTGGCACAATCAA TATCTCTTGAATATTGATTGTGCCAAACTGGGG-3'.

Transient transfections

GFP-LC3-expressing plasmids were transiently transfected into HCC cells as previously described [14].

Cell apoptosis assay

Apoptosis detection by Annexin V/PI and DAPI staining were performed as described [14]. 


\section{ROS examination}

To examine the accumulation of reactive oxygen species (ROS), cells were incubated with $10 \mu \mathrm{M}$ 5- and-6-chloromethyl-20,70dichlorodihydrofluorescein diacetate $\left(\mathrm{CM}-\mathrm{H}_{2} \mathrm{DCFDA} / \mathrm{DCFH}-\right.$ $\mathrm{DA}$; Invitrogen) for $30 \mathrm{~min}$ at $37^{\circ} \mathrm{C}$, respectively, followed by fluorescence microscopy.

In vivo studies

To establish a hepatocarcinoma xenograft tumor model, SMMC7721 and Hep3B cells $\left(2.0 \times 10^{7}\right.$ cells in $0.2 \mathrm{ml}$ serum-free DMEM medium) were injected subcutaneously into the right back of male athymic BALB/c nu/nu mice (5week-old). Tumor growth was monitored with electronic calipers using the formula: volume $=\mathrm{a} \times \mathrm{b}^{2} / 2$, where $a$ was the width at the widest point of the tumor, and $b$ was maximal width. When the tumors reached a mean tumor volume of $150-160 \mathrm{~mm}^{3}$, mice were randomly divided into five groups (each group had five mice) as follows: (a) control group (no treatment); (b) vehicle group ( $0.9 \%$ sodium chloride solution or AdSi-blank); (c) bevacizumab group; (d) autophagy inhibition (chloroquine or AdSi-Beclin1); (e) combination group. Mice received intraperitoneal injections of $5 \mathrm{mg} / \mathrm{kg}$ bevacizu$\mathrm{mab}$ or $60 \mathrm{mg} / \mathrm{kg} \mathrm{CQ}$ in $100 \mu \mathrm{l}$ of $0.9 \%$ sodium chloride solution, or were treated with AdSi-Beclin1 or AdSi-blank virus by way of multiple-center intratumoral injections of $50 \mu$ thrice weekly. All BALB/c nude mice were killed after 3 weeks of treatment.

\section{Statistical analysis}

Values were expressed as mean \pm SD. Statistical analysis between the two groups was calculated using Student's $t$ test, and analysis between multiple groups was calculated using the SPSS program, version 15.0. A $p<0.05$ was considered statistically significant.

\section{Results}

Effects of bevacizumab on xenograft tumors and autophagy

To evaluate the effect of antiangiogenesis on xenograft tumor growth, bevacizumab was used as an antigiogentic agent. As shown in Fig. 1a, after 21 days of treatment, the mean SMMC7721 xenograft tumor weight of bevacizumab treatment group was significantly reduced compared with that of the control $(1.16 \pm 0.15 \mathrm{~g}$ versus $1.61 \pm 0.28 \mathrm{~g} ; p<0.05)$ and vehicle (PBS) $(1.16 \pm 0.15 \mathrm{~g}$ versus $1.57 \pm 0.26 \mathrm{~g} ; p<0.05)$ groups (Fig. 1a). The mean tumor volume of the bevacizumab group was also markedly reduced compared with that of the control $\left(1,266.78 \pm 145.77 \mathrm{~mm}^{3}\right.$ versus $\left.1,695.63 \pm 194.74 \mathrm{~mm}^{3} ; p<0.05\right)$ and vehicle $\left(1,266.78 \pm 145.77 \mathrm{~mm}^{3}\right.$ versus $1,652.24 \pm$ $175.86 \mathrm{~mm}^{3} ; p<0.05$ ) groups (Fig. 1b). In Hep3B xenograft tumors, similar results were observed (Supplementary Fig. 3a,3b). Moreover, CD31 immunohistochemical staining showed lower microvessel density in the bevacizumab-treated tumor (Fig. 1c). Additionally, the higher expression of HIF-1 $\alpha$ in bevacizumab-treated tumors suggested that bevacizumab led to the enhancement of metabolic stress (Fig. 1c, d and Supplementary Fig. 3c).

Antiangiogenesis treatment leads to hypoxia and nutrient stress, both of which activate autophagy. Thus, we were interested in determining the effect of bevacizumab on autophagy in xenograft tumors. As shown in Fig. 1c and d, expression of Beclin1 and LC3 were upregulated in the bevacizumab group compared with the control and vehicle groups, which indicated activation of autophagy. Furthermore, electron microscopy analysis demonstrated an increase in autophagosomes in bevacizumab-treated tumors (Fig. 1e and Supplementary Fig. 3c). However, bevacizumab $(25 \mu \mathrm{g} / \mathrm{ml})$ could not activate autophagy in SMMC-7721 cells in vitro (Fig. 1f). Taken together, these results demonstrated that bevacizumab repressed tumor growth, reduced microvessel density, and induced autophagy in xenograft tumors.

Inhibition of autophagy increases apoptosis of hepatocarcinoma cells during nutrient starvation and hypoxia in vitro

Reducing tumor vasculature by antiangiogenic agents leads to nutrient starvation and hypoxia. These conditions of nutrient deficiency activate autophagy. To evaluate the effect of autophagy on nutrient deficiency in HCC cells, nutrientdeprived medium and hypoxia were used to mimic the nutrient-deficient condition, which tumor cells were exposed to in vivo during bevacizumab treatment. First, SMMC7721 cells, transfected with a GFP-LC3 plasmid, were cultured in nutrient-starved medium. As shown in Fig. 2a, nutrientstarved SMMC7721 cells had a significantly increased number of GFP-LC3 dots, an indicator of autophagy activation. This was enhanced by CQ treatment and attenuated by 3-MA. The results of WTS-8 assay showed that 3-MA or CQ treatment led to an increase in cell death (Fig. 2b). Cell morphology detection demonstrated that typical apoptotic changes appeared in 3-MA or CQ-treated nutrient-starved cells, including marked rounding, shrinkage, and detachment of cells from the culture dish (Fig. 2c). The results of DAPI and Annexin V/PI staining also demonstrated that treatment with autophagy inhibitor in nutrient-starved SMMC7721 cells induced significantly more apoptosis (Fig. 2d, e). The same results were also observed in Hep3B cells (Fig. 3). Taken together, these data suggest that inhibition of autophagy results in increased apoptosis in HCC cells during nutrient starvation. 


\section{a}
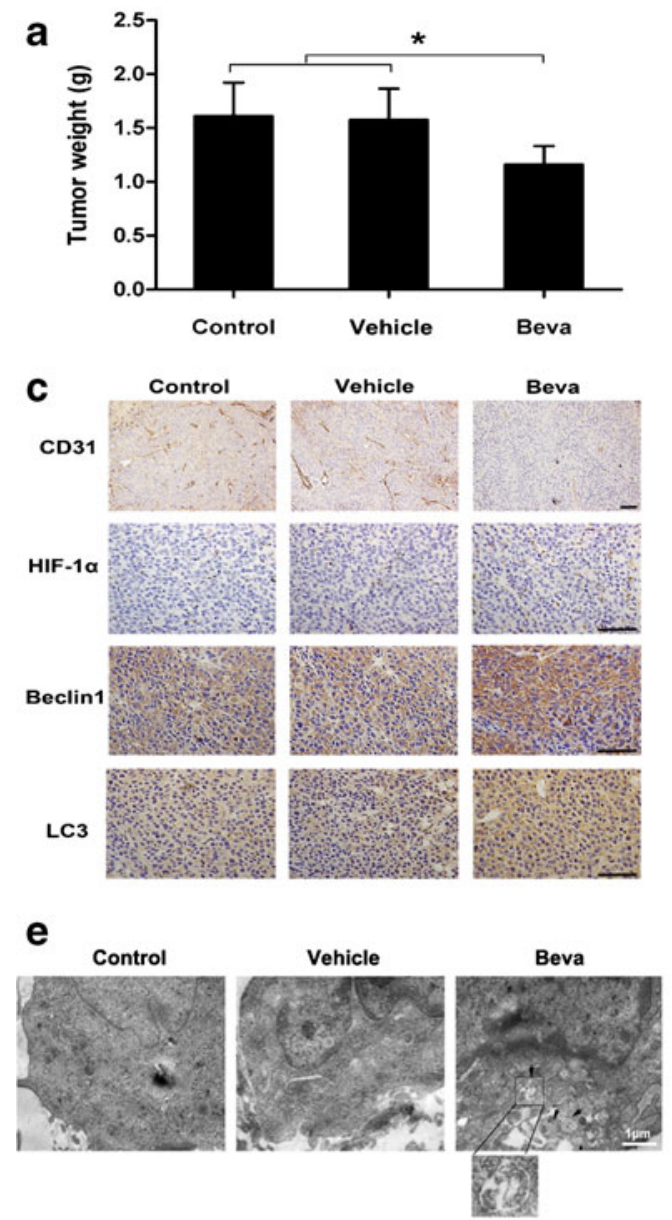

b

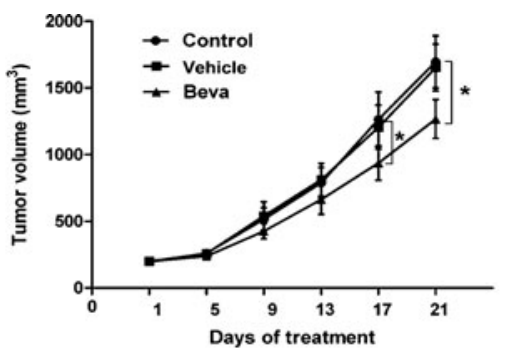

d
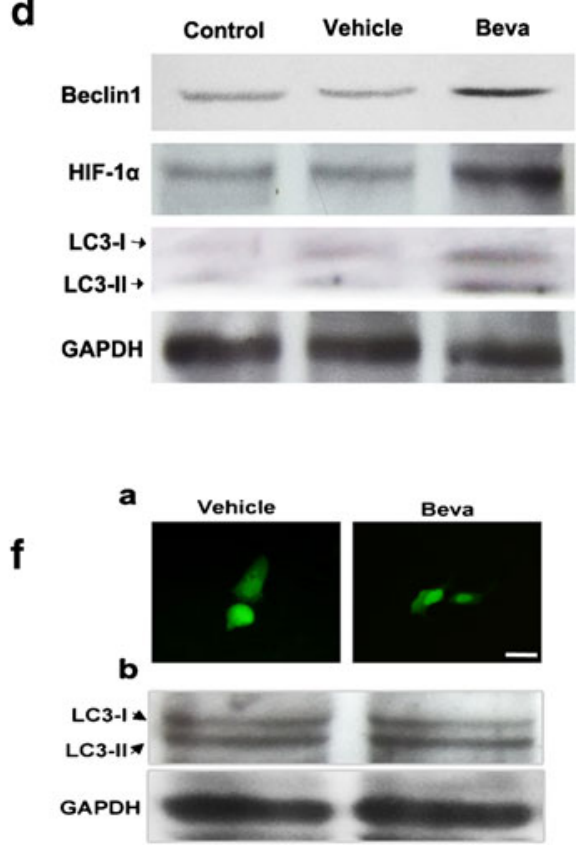

Fig. 1 Bevacizumab inhibits tumor growth and induces autophagy in SMMC-7721 xenograft model. The tumor models are described in the "Materials and methods" section. a After 21 days of treatment, the tumor xenografts were excised, and tumor weights were measured. Data (mean \pm SD) represent the mean of at least three independent experiments. b Tumor progression was evaluated by measuring tumor volume every 4 days. c Immunohistochemical staining of CD31, Beclin1, LC3, and HIF-1 $\alpha$. d Whole cell lysates of SMMC-7721

To further confirm the role of the autophagic machinery, we used siRNA to inhibit the Beclin1 gene, which is essential for autophagosome generation [16]. As shown in Supplementary Fig. 1, silencing of Beclin1 markedly increased nutrient starvation-induced apoptosis in HCC cells. Together, these data strongly indicate that $\mathrm{HCC}$ cells resist nutrient starvation-induced apoptosis by activating autophagy.

To test the protective role of autophagy on HCC cells during hypoxia, SMMC7721 and Hep3B cells, transfected with either no-target siRNA or Beclin1 siRNA, were cultured under normoxia $\left(20 \% \mathrm{O}_{2}\right)$ or hypoxia $\left(1 \% \mathrm{O}_{2}\right)$ for $36 \mathrm{~h}$. As shown in Fig. 4a, d, and e, hypoxic HCC cells showed an increase in the formation of GFP-LC3 dots, which was markedly inhibited by siBeclin1. Moreover, siBeclin1 treatment significantly increased apoptosis of hypoxic HCC cells (Fig. 4b, c, f, g). Thus, our data demonstrated that autophagy promoted HCC cell survival under hypoxic conditions. xenograft tumors were subjected to Western blot analysis for Beclin1, LC3, and HIF-1 $\alpha$. GAPDH expression was used as a loading control (scale bar, $100 \mu \mathrm{m}$ ). Beva: bevacizumab. e Representative electron microscopic images are shown. An arrow indicates the autophagosome. f SMMC-7721 cells were transfected with a GFP-LC3 plasmid and were then treated with vehicle and bevacizumab $(25 \mu \mathrm{g} / \mathrm{ml})$ for $24 \mathrm{~h}$ (scale bar, $50 \mu \mathrm{m}$ ). Representative images are shown (a). Whole cell lysates were subject to Western blotting for LC3 (b)

Inhibition of autophagy promotes the anticancer effects of bevacizumab in HCC xenograft tumors

To investigate the in vivo efficacy of the combined treatment of an autophagy inhibitor and angiogenesis inhibitor in hepatocarcinoma, a SMMC-7721 hepatocarcinoma xenograft tumor model was established in nude mice. As shown in Fig. 5a, the increased accumulation of LC3 and formation of the autophagosome were observed in the CQ and bevacizumab combination treatment group. These data suggest that CQ can successfully inhibit autophagy in vivo. The results of CD31 staining also suggested that $\mathrm{CQ}$ had no effect on angiogensis in xenograft tumors. Furthermore, there were no significant differences in the body weight of nude mice between groups (Fig. 5b). After 21 days of treatment, the mean tumor volume of the combination group was significantly reduced compared with the bevacizumab group $\left(818.68 \pm 152.52 \mathrm{~mm}^{3}\right.$ versus 
Fig. 2 Autophagy inhibition by 3-MA or CQ promotes apoptosis of SMMC-7721 cells during nutrient starvation. SMMC7721 cells were cultured in nutrient-starved medium and treated with CQ $(20 \mathrm{mM})$ or 3MA $(10 \mathrm{mM})$ for $24 \mathrm{~h}$. a After being transfected with GFPtagged LC3 for $24 \mathrm{~h}$, SMMC7721 cells were treated as mentioned above, followed by fluorescence microscopy (left side, scale bar, $50 \mu \mathrm{m})$. The right side is quantitative analysis of GFP-LC3 punctate dots/ cell. b Cell viability was determined by a WST- 8 assay. c Cell morphology is shown (scale bar, $50 \mu \mathrm{m})$. d Apoptotic cells were captured by DAPI staining of the condensed and fragmented nuclei. The arrow indicates apoptotic cells (left side, scale bar, $50 \mu \mathrm{m})$. The right side is quantitative analysis of apoptotic cells. e Analysis of Annexin-V and PI staining. The Annexin $\mathrm{V}^{+} / \mathrm{PI}^{-}$or Annexin $\mathrm{V}^{+}$/ $\mathrm{PI}^{+}$cells were considered apoptotic cells. Data of three replicates are shown as means \pm SD. $*(p<0.05), * *(p<0.01)$, $* * *(p<0.001) . F N$ : full nutrient, $N F$ : nutrient-free

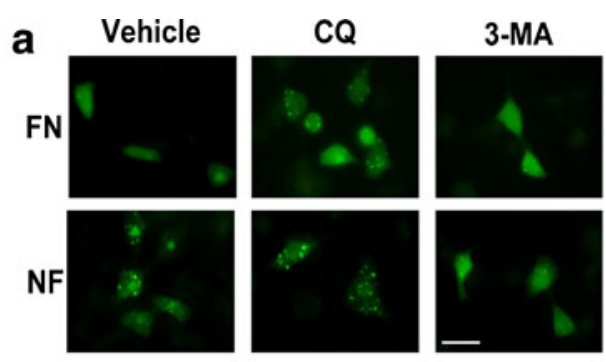

b

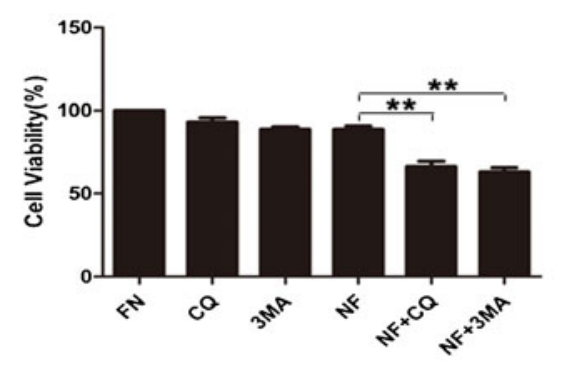

C
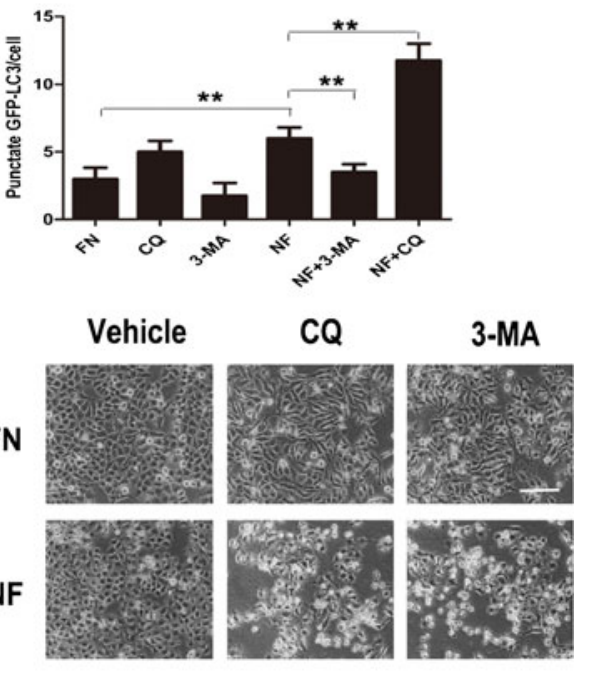

d

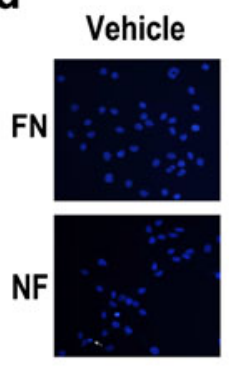

CQ

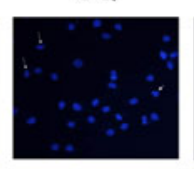

3-MA
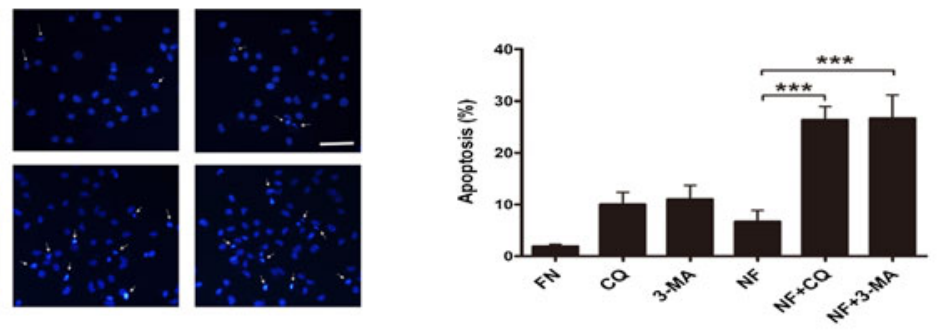

FN

NF

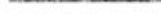

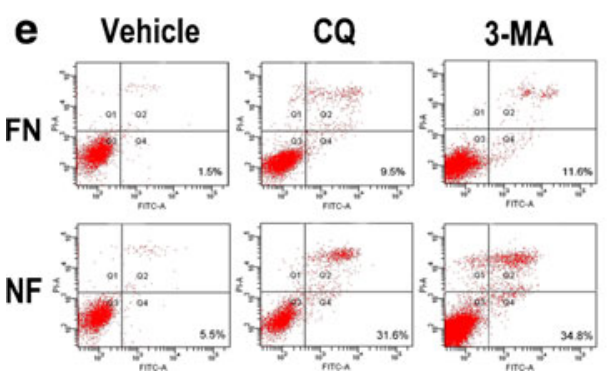

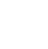


Fig. 3 3-MA promotes the apoptosis of Hep3B cells during nutrient starvation. Hep3B cells were cultured in nutrientstarved medium and treated with 3-MA $(10 \mathrm{mM})$ for $24 \mathrm{~h}$. a After transfection with GFPtagged LC3 for $24 \mathrm{~h}$, Hep3B cells were treated as mentioned above, and images were taken under a fluorescence microscope(scale bar, $50 \mu \mathrm{m}$ ). b Cell morphology is shown (scale bar, $50 \mu \mathrm{m}$ ). c Apoptotic cells were captured by DAPI staining of the condensed and fragmented nuclei. The arrow indicates apoptotic cells (scale bar, $50 \mu \mathrm{m})$. d Analysis of Annexin$\mathrm{V}$ and PI staining. Annexin $\mathrm{V}^{+} /$ $\mathrm{PI}^{-}$or Annexin $\mathrm{V}^{+} / \mathrm{PI}^{+}$cells were considered apoptotic cells. e Quantitative analysis of GFPLC3 punctate dots/cell of $\mathbf{a} . \mathbf{f}$ Cell viability was determined by a WST-8 assay. (g) Quantitative analysis of apoptotic cells of $\mathbf{d}$. Data of three replicates are shown as mean $\pm \mathrm{SD}$. $* *(p<$ $0.01),{ }^{* * *}(p<0.001) . F N$ : full nutrient, $N F$ : nutrient-free
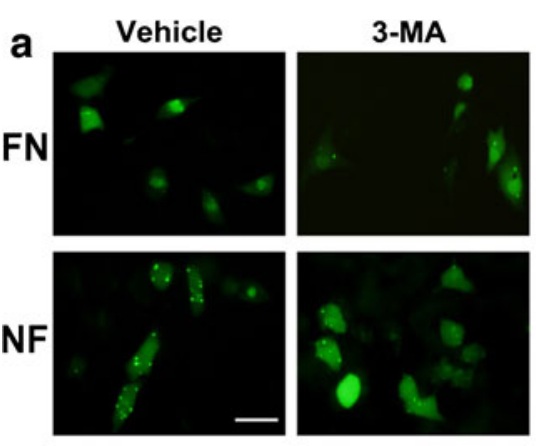

b
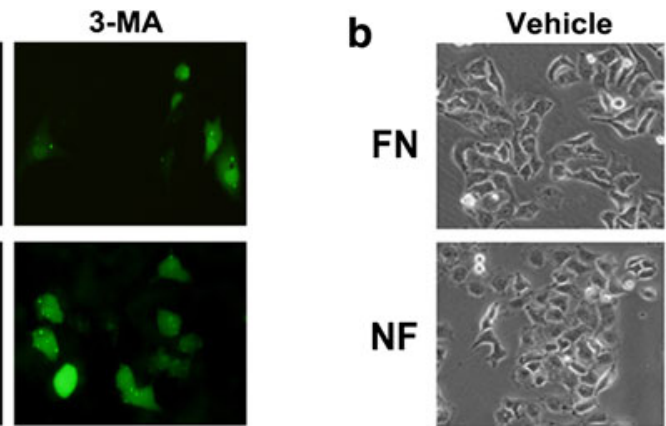

C Vehicle

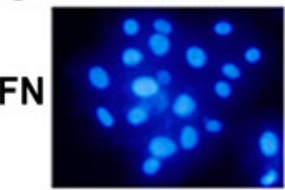

3-MA

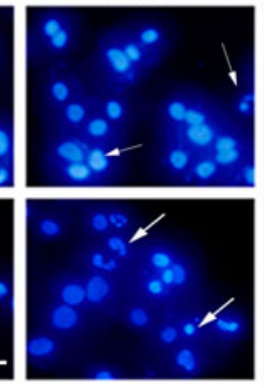

d

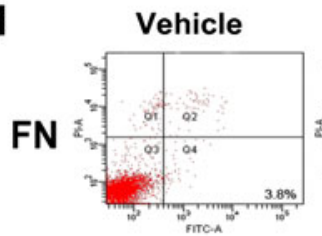

3-MA
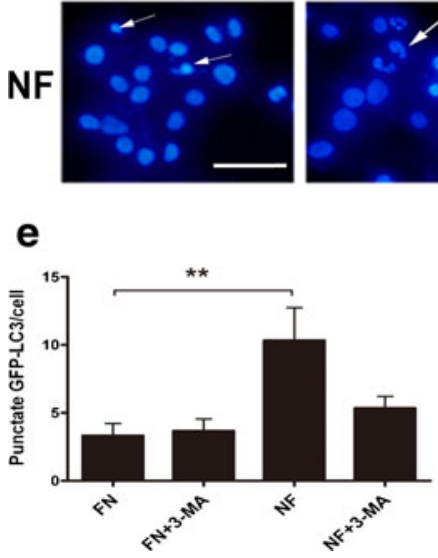

f
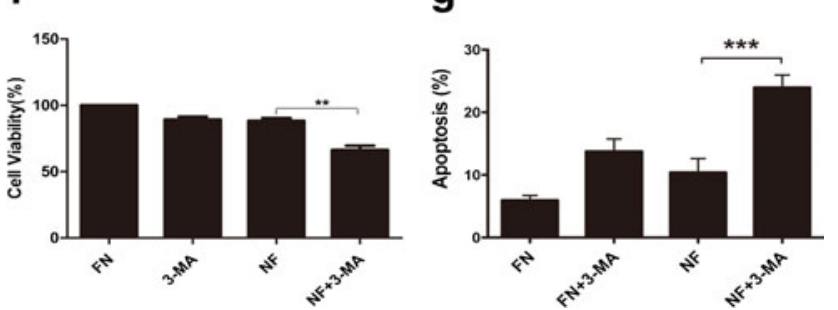

xenograft tumor model, combined treatment with bevacizumab and chloroquine also impaired tumor cell proliferation and increased intratumoral apoptosis (Supplementary Fig. 3c-3e). These results suggest that impaired cell proliferation and increased cell apoptosis contribute to the tumor-suppressive function of bevacizumab and CQ combination treatment.

ROS generation is enhanced and contributes to cell death in nutrient-deficient HCC cells during autophagy inhibition

Metabolic stress causes ROS accumulation and increased ROS leads to cell death. To determine the role of ROS in cell death of nutrient-deficient HCC cells after autophagy inhibition, we first detected whether autophagy could modulate ROS generation in nutrient-deficient cells. Intracellular levels of ROS in HCC cells following treatment with $\mathrm{CQ}$, nutrient-starved medium, hypoxia, or the combination for indicated time (nutrient starvation for $24 \mathrm{~h}$; hypoxia for $36 \mathrm{~h}$ ) were examined. In both SMMC-7721 and Hep3B cell lines, there were marked increases in the ROS levels after treatment with CQ and nutrient starvation or hypoxia when compared with cells under nutrient starvation, hypoxia, or CQ treatment alone (Fig. 7a,e). To evaluate whether enhanced ROS levels may contribute to the cell death of nutrient-deficient
HCC cells with autophagy inhibition, we applied the antioxidant NAC to eliminate ROS. SMMC-7721 and Hep3B cells pretreated with NAC displayed significantly reduced cell death with CQ and nutrient starvation or hypoxia combined treatment (Fig. 7b, c, f, and g). Thus, increased ROS levels have an important role in the induction of cell death by nutrient deficiency in combination with autophagy inhibitor. We also examined the immunostaining of 8-hydroxydeoxyguanosine (8$\mathrm{OHdG}$ ) in xenograft tumor tissue, as $8-\mathrm{OHdG}$ is an indicator of DNA oxidative damage. As shown in Fig. 7d and h, more 8OHdG-positive cells were observed in the bevacizumab and CQ cotreatment group. Together, these results suggest that autophagy inhibition enhances metabolic stress-induced oxidative damage, which contributes to the death of nutrient-starved HCC cells.

\section{Discussion}

We report here that bevacizumab treatment inhibits the growth of xenograft tumors and activates autophagy by reducing the formation of new blood vessels. The combination of autophagy inhibitor and bevacizumab treatment further inhibited 


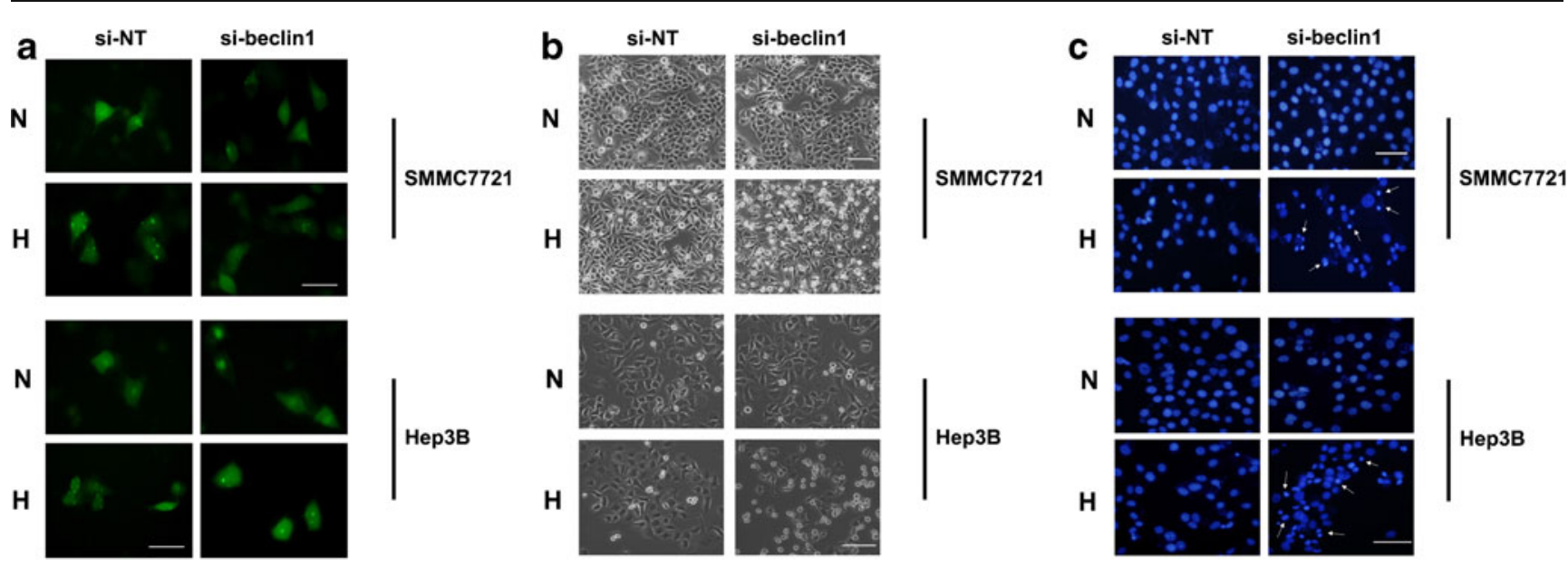

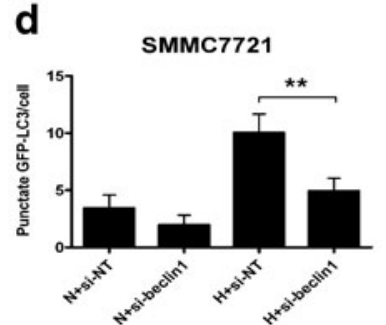

e

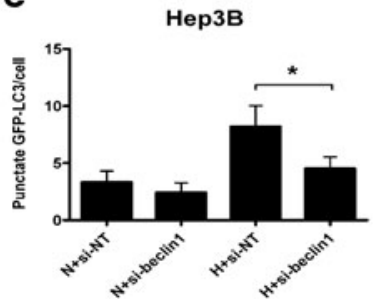

f

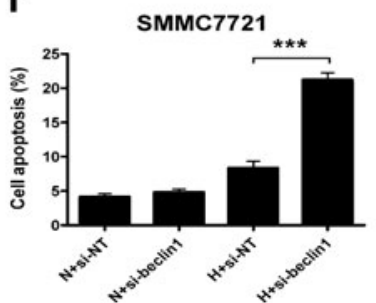

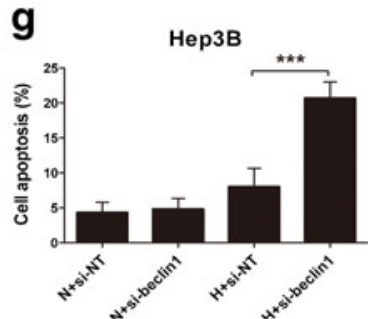

Fig. 4 SiBeclin1 reduces cell viability and promotes apoptosis of HCC cells during hypoxic stress. After transfection with the siBeclin1 for 1 day, SMMC-7721 and Hep3B cells were cultured under normoxia $\left(20 \% \mathrm{O}_{2}\right)$ or hypoxia $\left(1 \% \mathrm{O}_{2}\right)$ for $36 \mathrm{~h}$. a $\mathrm{HCC}$ cells were transfected with a GFP-tagged LC3 plasmid, and images were taken under a fluorescence microscope (scale bar, $50 \mu \mathrm{m}$ ). b Cell morphology was captured by a light microscope (scale bar, $50 \mu \mathrm{m}$ ). c Apoptotic cells were detected by DAPI staining. Arrows indicate the apoptotic cell (scale bar, $50 \mu \mathrm{m}$ ). d, e Quantitative analysis of GFP-LC3 punctate dots/cell of a. f, $\mathbf{g}$ Quantitative analysis of apoptotic cells of $\mathbf{c}$. Data represent the mean of three independent experiments and are shown as mean \pm SD. $*(p<0.05), * *(p<0.01), * * *(p<0.001)$. si-NT: no target siRNA. $N$ : normoxia, $H$ : hypoxia

Antiangiogenic strategy deprives tumors of their blood supply by reducing the tumor vasculature, which leads to the induction of necrosis or apoptosis in tumor cells, ultimately inducing tumor regression [18]. In this study, bevacizumab treatment inhibited the growth of tumor xenografts and activated autophagy by reducing blood vessel generation. However, bevacizumab treatment was not able to induce autophagy in HCC cells in vitro. Autophagy can be activated by metabolic stress[19]. Thus, the
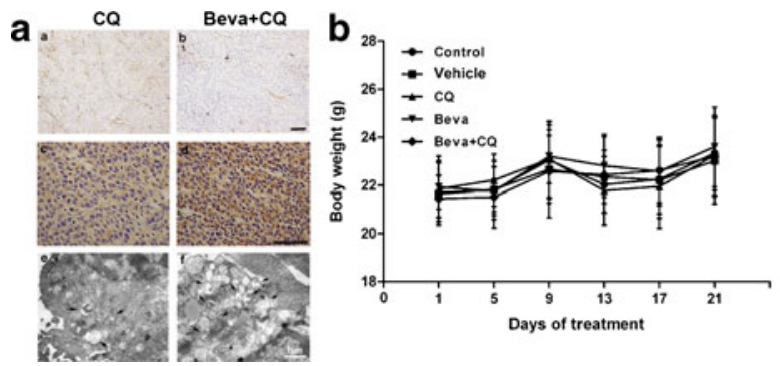

Fig. 5 Therapeutic efficacy of bevacizumab was enhanced by CQ in hepatocarcinoma xenograft model. Tumor models were established. a SMMC-7721 tumor xenografts were treated with CQ or a combination of CQ and bevacizumab. Immunohistochemical staining for CD31 (a and b) and LC3 (c and d) was detected. Representative electron microscopic images of autophagosome (e and $\mathbf{f}$ ) are shown. An arrow
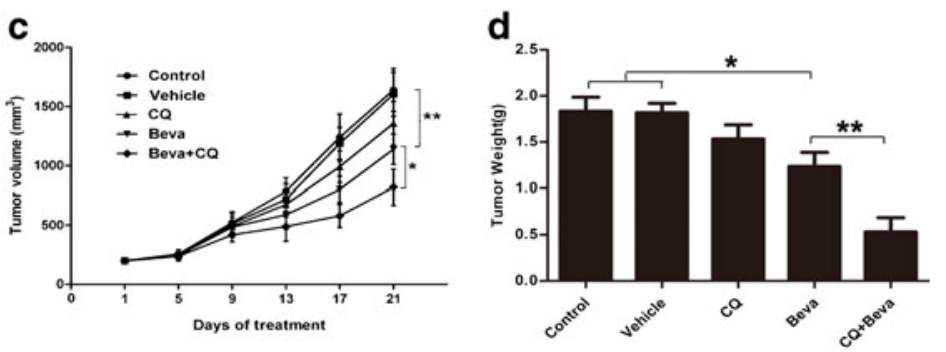

indicates the autophagosome (scale bar, $100 \mu \mathrm{m}$ ). b Body weights of all mice were measured every 4 days. c Tumor progression was evaluated by measuring tumor volume every 4 days. d After 21 days of treatment, mice were sacrificed, and tumor weights were measured. Data represent the mean of three independent experiments and are shown as mean \pm SD. $*(p<0.05), * *(p<0.01)$. Beva: bevacizumab 
Control

a

PCNA

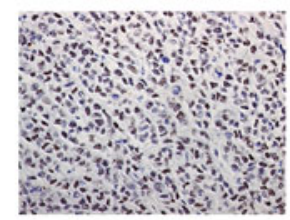

b

Actived

caspase-3

C

TUNEL

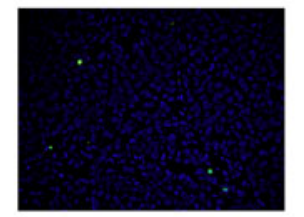

d

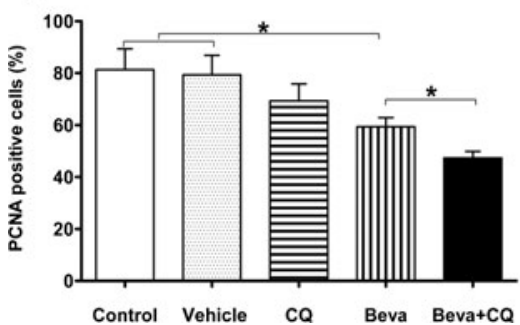

Fig. 6 Combination treatment of bevacizumab and CQ leads to reduced cell proliferation and increased apoptosis in a hepatocarcinoma xenograft tumor model. a, b Immunohistochemical staining of representative tumor tissue samples with PCNA and activated caspase-3 antibodies(scale bar, $100 \mu \mathrm{m}$ ). c Apoptosis of tumor tissues was

upregulation of autophagy during bevacizumab treatment is a response of xenograft tumor cells to metabolic stress.

Under nutrient deficiency, autophagy is induced and promotes tumor cell survival $[20,21]$. Consistent with this, we demonstrated here that autophagy protected HCC cells from nutrient starvation and hypoxia-induced apoptosis in vitro. Interestingly, early reports have shown that autophagy activated by hypoxia can mediate the tolerance of hepatocellular carcinoma cells to nutrient deprivation [22]. Autophagy promotes cell survival in various ways, such as providing energy substrates, mitigating accumulated ROS, and reducing damaged organelles [20]. In fact, in the early or later stages of tumor progression, autophagy is activated in metabolically stressed tumor regions [23]. These findings suggest that maintaining cell survival by autophagy in unfavorable conditions is essential for tumor development. Thus, we suspect that inhibition of autophagy may be an effective way of improving antiangiogenic strategy in vivo. In this study, we observed that the combined treatment of bevacizumab and CQ led to dramatic tumor repression. These results indicate that inhibition of autophagy impairs the survival mechanism of tumors and
CQ
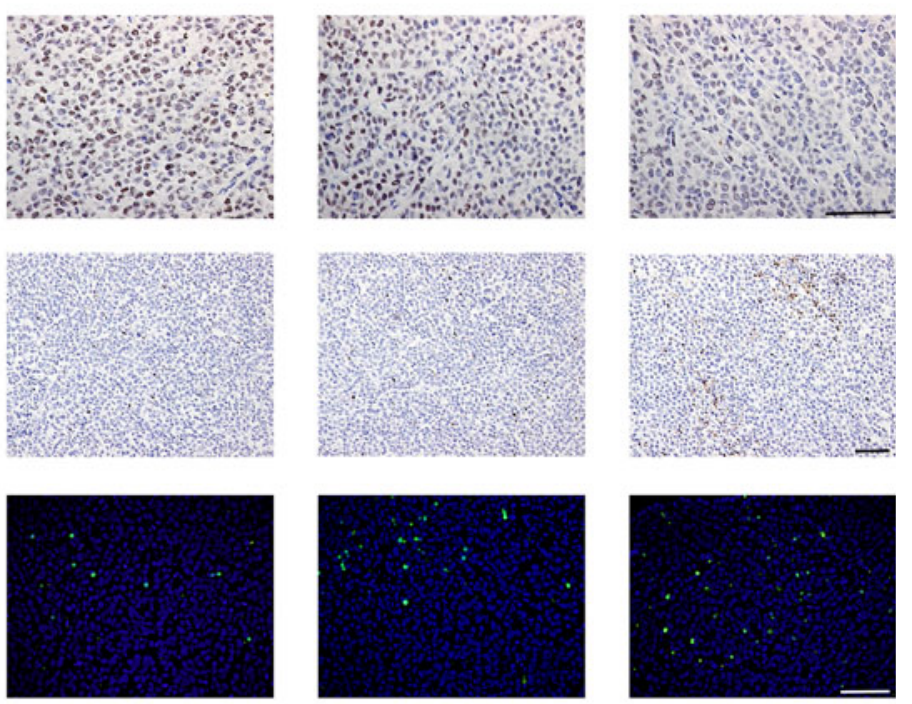

f

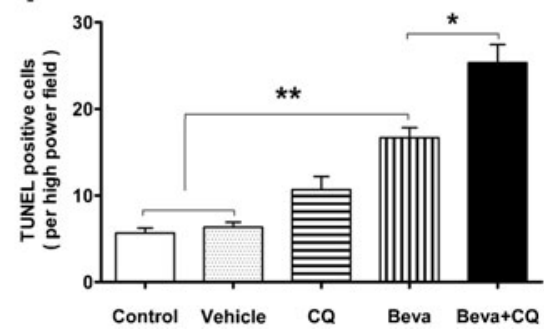

measured by TUNEL assays; green nucleus shows TUNEL-positive cells(scale bar, $100 \mu \mathrm{m})$. d, e Quantitative analysis of proliferative and caspase-3-activated cells. f Quantitative analysis of TUNEL-positive cells. Data represent three independent experiments shown as mean \pm SD. $*(p<0.05), * *(p<0.01)$. Beva: bevacizumab

intensifies metabolic stress, consequently leading to tumor inhibition. Indeed, previous studies have shown that endothelial cells initiate autophagy as a survival mechanism against angiogenesis inhibitor-induced apoptosis [24, 25]. These findings further strengthen our hypothesis that autophagy inhibition can enhance the therapeutic efficacy of antiangiogenic strategy. In this study, we showed that the enhancement of apoptosis and impairment of cell proliferation are most likely the mechanisms underlying the antitumor effects of combined bevacizumab and CQ treatment. Meanwhile, inhibition of autophagy may impair its function of preserving organelle required for cell growth [20] and render cell cycle arrest [26].

It was suggested that low concentrations of ROS promoted cell proliferation [27], but high ROS concentrations lead to cell death [28]. Therefore, modulation of intracellular ROS levels is critical for cancer cell survival, as metabolic stress causes ROS accumulation [29, 30]. In this study, we observed a modest increase in cellular ROS generation in nutrient-starved and hypoxic HCC cells. However, the combination of CQ treatment and nutrient starvation or hypoxia resulted in a marked increase in ROS generation. Increased levels of ROS contributed to cell 

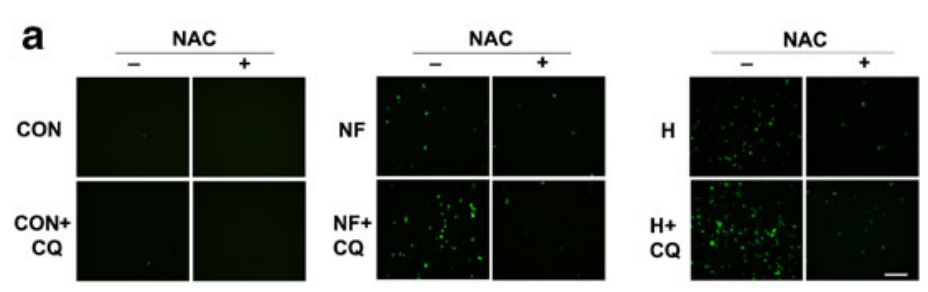

b

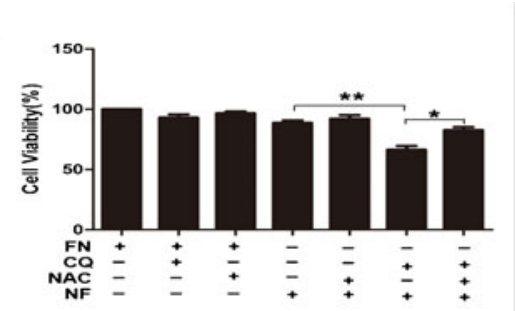

C

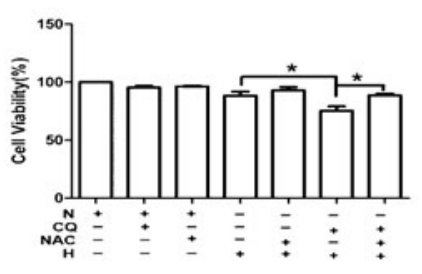

d

SMMC7721

e
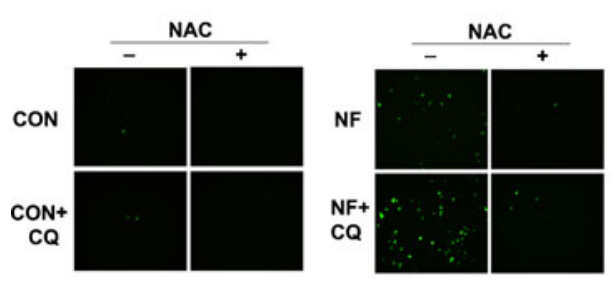

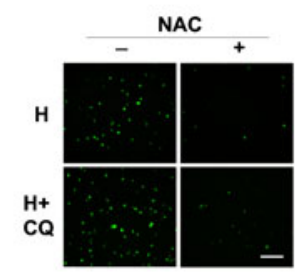

f

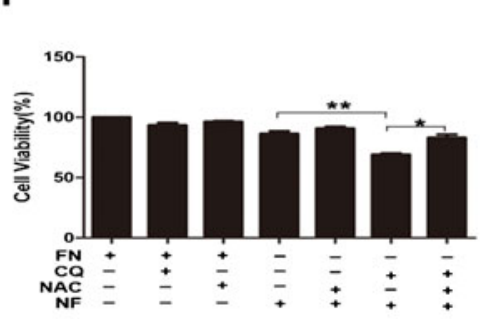

g

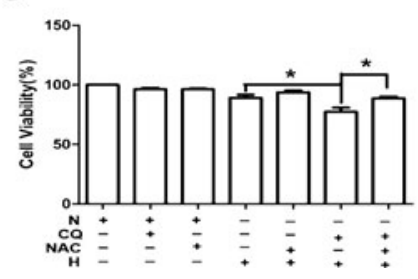

Fig. 7 ROS generation is augmented and contributes to cell death in nutrient-deficient HCC cells when autophagy is inhibited. SMMC7721 and Hep3B cells were incubated in nutrient-starved medium for $24 \mathrm{~h}$ or hypoxia for $36 \mathrm{~h}$ with $10 \mu \mathrm{M}$ CQ. a, e Cellular ROS generation was determined using DCF-DA staining (scale bar, $100 \mu \mathrm{m}$ ). b, c, f, $\mathbf{g}$ Cells were pretreated with $10 \mathrm{mM} \mathrm{NAC}$ for $2 \mathrm{~h}$; then cells were incubated in EBSS medium or hypoxia alone or with $10 \mu \mathrm{M}$ CQ for the indicated time. Cell viability was determined by a WST- 8 assay. Data shown are mean $\pm \mathrm{SD}$ from at least three independent experiments. d, h Immunohistochemical staining of 8-OhdG (scale bar, $100 \mu \mathrm{m}$ ). $F N$ : full nutrient, $N F$ : nutrient-free, $N$ : normoxia, $H$ : hypoxia death in nutrient-starved or hypoxic HCC cells when autophagy was inhibited, but treatment with the NAC antioxidant markedly reduced this phenonemon. Consistent with our findings, a recent study suggested that induction of autophagy could decrease ROS levels and moderate ROS-induced cell death [30]. The removal of oxidatively damaged organelles and proteins by autophagy provides a second level of protection against oxidative stress [31]. However, the interaction between ROS and autophagy is complex. ROS, especially mitochondrial ROS, serve as signaling molecules in inducing autophagy [32, 33].

Although angiogenesis inhibitors were considered as desirable anticancer agents, it was found that most tumors were still resistant to antiangiogenesis [34, 35].
The mechanisms of resistance to antiangiogenesis are still unknown [34, 35]. In this study, we provide compelling data suggesting that inhibition of autophagy can enhance the tumor-suppressive function of bevacizumab. Thus, activation of autophagy may be involved in resistance to antiangiogenic strategies. Autophagy may help tumor cells mitigate metabolic stress and promote cell survival during antiangiogenesis, which may provide tumor cells with a greater change to develop other ways to resist antiangiogentic therapy. In conclusion, we show that inhibition of autophagy may be a novel way to increase the efficicacy of antiangiogenic agents in the treatment of $\mathrm{HCC}$. 
Acknowledgments This project was supported by National Natural Science Foundation of China (grant numbers: 81030041, 30901722, 31171321, 81000970, 81101622, 81201584, 30921006); Key Basic Research Project of China (grant numbers: 2012CBA01303, 2011CB966200, 2010CB945600); Special Funds for National Key Sci-Tech Special Project of China (grant numbers: 2012ZX10002011, 2012ZX10002016); and Shanghai Science and Technology Committee (grant numbers:12ZR1454200, 12ZR1439800, 11ZR1449500, 10ZR1439600, 10411963100, 10ZR1439900).

Disclosure statement The authors declare no conflict of interest.

Open Access This article is distributed under the terms of the Creative Commons Attribution License which permits any use, distribution, and reproduction in any medium, provided the original author(s) and the source are credited.

\section{References}

1. Zhu AX, Duda DG, Sahani DV, Jain RK (2011) HCC and angiogenesis: possible targets and future directions. Nat Rev Clin Oncol 8:292-301

2. von Marschall Z, Cramer T, Hocker M, Finkenzeller G, Wiedenmann B, Rosewicz S (2001) Dual mechanism of vascular endothelial growth factor upregulation by hypoxia in human hepatocellular carcinoma. Gut 48:87-96

3. Moon WS, Rhyu KH, Kang MJ, Lee DG, Yu HC, Yeum JH, Koh GY, Tarnawski AS (2003) Overexpression of VEGF and angiopoietin 2: a key to high vascularity of hepatocellular carcinoma? Mod Pathol 16:552-557

4. Presta LG, Chen H, O'Connor SJ, Chisholm V, Meng YG, Krummen L, Winkler M, Ferrara N (1997) Humanization of an anti-vascular endothelial growth factor monoclonal antibody for the therapy of solid tumors and other disorders. Cancer Res 57:45934599

5. Jain RK (2005) Normalization of tumor vasculature: an emerging concept in antiangiogenic therapy. Science 307:58-62

6. Finn RS, Zhu AX (2009) Targeting angiogenesis in hepatocellular carcinoma: focus on VEGF and bevacizumab. Expert Rev Anticancer Ther 9:503-509

7. Xie Z, Klionsky DJ (2007) Autophagosome formation: core machinery and adaptations. Nat Cell Biol 9:1102-1109

8. Kuma A, Hatano M, Matsui M, Yamamoto A, Nakaya H, Yoshimori T, Ohsumi Y, Tokuhisa T, Mizushima N (2004) The role of autophagy during the early neonatal starvation period. Nature 432:1032-1036

9. Klionsky DJ (2007) Autophagy: from phenomenology to molecular understanding in less than a decade. Nat Rev Mol Cell Biol 8:931-937

10. Rubinsztein DC, Gestwicki JE, Murphy LO, Klionsky DJ (2007) Potential therapeutic applications of autophagy. Nat Rev Drug Discov 6:304-312

11. Klionsky DJ, Abeliovich H, Agostinis P, Agrawal DK, Aliev G, Askew DS, Baba M, Baehrecke EH, Bahr BA, Ballabio A et al (2008) Guidelines for the use and interpretation of assays for monitoring autophagy in higher eukaryotes. Autophagy 4:151-175

12. Yang PM, Liu YL, Lin YC, Shun CT, Wu MS, Chen CC (2010) Inhibition of autophagy enhances anticancer effects of atorvastatin in digestive malignancies. Cancer Res 70:7699-7709

13. Takasu H, Sugita A, Uchiyama Y, Katagiri N, Okazaki M, Ogata E, Ikeda K (2006) c-Fos protein as a target of anti-osteoclastogenic action of vitamin $\mathrm{D}$, and synthesis of new analogs. $\mathrm{J}$ Clin Invest 116:528-535

14. Song J, Qu Z, Guo X, Zhao Q, Zhao X, Gao L, Sun K, Shen F, Wu M, Wei L (2009) Hypoxia-induced autophagy contributes to the chemoresistance of hepatocellular carcinoma cells. Autophagy 5:1131-1144

15. Guo X, Wang W, Zhou F, Lu Z, Fang R, Jia F, Bu X, Li R, Zhang $\mathrm{B}, \mathrm{Wu} \mathrm{M}$ et al (2008) siRNA-mediated inhibition of hTERT enhances chemosensitivity of hepatocellular carcinoma. Cancer Biol Ther 7:1555-1560

16. Cao Y, Klionsky DJ (2007) Physiological functions of Atg6/Beclin 1: a unique autophagy-related protein. Cell Res 17:839-849

17. Nelson DA, Tan TT, Rabson AB, Anderson D, Degenhardt K, White E (2004) Hypoxia and defective apoptosis drive genomic instability and tumorigenesis. Genes Dev 18:20952107

18. Huang KW, Wu HL, Lin HL, Liang PC, Chen PJ, Chen SH, Lee HI, Su PY, Wu WH, Lee PH et al (2010) Combining antiangiogenic therapy with immunotherapy exerts better therapeutical effects on large tumors in a woodchuck hepatoma model. Proc Natl Acad Sci U S A 107:14769-14774

19. Tsuchihara K, Fujii S, Esumi H (2009) Autophagy and cancer: dynamism of the metabolism of tumor cells and tissues. Cancer Lett 278:130-138

20. Guo JY, Chen HY, Mathew R, Fan J, Strohecker AM, Karsli-Uzunbas G, Kamphorst JJ, Chen G, Lemons JM, Karantza V et al (2011) Activated Ras requires autophagy to maintain oxidative metabolism and tumorigenesis. Genes Dev 25:460-470

21. Sato K, Tsuchihara K, Fujii S, Sugiyama M, Goya T, Atomi Y, Ueno T, Ochiai A, Esumi H (2007) Autophagy is activated in colorectal cancer cells and contributes to the tolerance to nutrient deprivation. Cancer Res 67:9677-9684

22. Song J, Guo X, Xie X, Zhao X, Li D, Deng W, Song Y, Shen F, Wu M, Wei L (2011) Autophagy in hypoxia protects cancer cells against apoptosis induced by nutrient deprivation through a Beclin1-dependent way in hepatocellular carcinoma. J Cell Biochem 112:3406-3420

23. Degenhardt K, Mathew R, Beaudoin B, Bray K, Anderson D, Chen G, Mukherjee C, Shi Y, Gelinas C, Fan Y et al (2006) Autophagy promotes tumor cell survival and restricts necrosis, inflammation, and tumorigenesis. Cancer Cell 10:51-64

24. Nguyen TM, Subramanian IV, Xiao X, Ghosh G, Nguyen P, Kelekar A, Ramakrishnan S (2009) Endostatin induces autophagy in endothelial cells by modulating Beclin 1 and beta-catenin levels. J Cell Mol Med 13:3687-3698

25. Nguyen TM, Subramanian IV, Kelekar A, Ramakrishnan S (2007) Kringle 5 of human plasminogen, an angiogenesis inhibitor, induces both autophagy and apoptotic death in endothelial cells. Blood 109:4793-4802

26. Altman BJ, Jacobs SR, Mason EF, Michalek RD, MacIntyre AN, Coloff JL, Ilkayeva O, Jia W, He YW, Rathmell JC (2011) Autophagy is essential to suppress cell stress and to allow BCR-Ablmediated leukemogenesis. Oncogene 30:1855-1867

27. Fratelli M, Goodwin LO, Orom UA, Lombardi S, Tonelli R, Mengozzi M, Ghezzi P (2005) Gene expression profiling reveals a signaling role of glutathione in redox regulation. Proc Natl Acad Sci U S A 102:13998-14003

28. Wellen KE, Thompson CB (2010) Cellular metabolic stress: considering how cells respond to nutrient excess. Mol Cell 40:323332

29. Azad MB, Chen Y, Gibson SB (2009) Regulation of autophagy by reactive oxygen species (ROS): implications for cancer progression and treatment. Antioxid Redox Signal 11:777-790

30. Bensaad K, Cheung EC, Vousden KH (2009) Modulation of intracellular ROS levels by TIGAR controls autophagy. EMBO J 28:3015-3026 
31. Moore MN (2008) Autophagy as a second level protective process in conferring resistance to environmentally-induced oxidative stress. Autophagy 4:254-256

32. Scherz-Shouval R, Shvets E, Fass E, Shorer H, Gil L, Elazar Z (2007) Reactive oxygen species are essential for autophagy and specifically regulate the activity of Atg4. EMBO J 26:1749-1760
33. Chen Y, Azad MB, Gibson SB (2009) Superoxide is the major reactive oxygen species regulating autophagy. Cell Death Differ 16:1040-1052

34. Azam F, Mehta S, Harris AL (2010) Mechanisms of resistance to antiangiogenesis therapy. Eur J Cancer 46:1323-1332

35. Eikesdal HP, Kalluri R (2009) Drug resistance associated with antiangiogenesis therapy. Semin Cancer Biol 19:310-317 